\title{
\begin{tabular}{l|l} 
Mitraries & DSpace@MIT
\end{tabular}
}

\author{
MIT Open Access Articles
}

\section{Total Synthesis and Anti-Cancer Activity of All Known Communesin Alkaloids and Related Derivatives}

The MIT Faculty has made this article openly available. Please share how this access benefits you. Your story matters.

Citation: Pompeo, Matthew M. "Total Synthesis and Anti-Cancer Activity of All Known Communesin Alkaloids and Related Derivatives." Journal of the American Chemical Society 141, 36 (August 2019): 14411-14420 (C) 2019 American Chemical Society

As Published: http://dx.doi.org/10.1021/jacs.9b07397

Publisher: American Chemical Society (ACS)

Persistent URL: https://hdl.handle.net/1721.1/122288

Version: Final published version: final published article, as it appeared in a journal, conference proceedings, or other formally published context

Terms of use: Creative Commons Attribution-NonCommercial-NoDerivs License 


\title{
Total Synthesis and Anti-Cancer Activity of All Known Communesin Alkaloids and Related Derivatives
}

\author{
Matthew M. Pompeo, ${ }^{\dagger}$ J Jaime H. Cheah, ${ }^{\ddagger}$ and Mohammad Movassaghi ${ }^{*}{ }^{\dagger}$ \\ ${ }^{\dagger}$ Department of Chemistry, Massachusetts Institute of Technology, Cambridge, Massachusetts 02139, United States \\ ${ }^{\ddagger}$ The Koch Institute for Integrative Cancer Research, Massachusetts Institute of Technology, 500 Main Street, Cambridge, \\ Massachusetts 02139, United States
}

Supporting Information

ABSTRACT: A unified enantioselective total synthesis and anticancer evaluation of all known epoxide-containing communesin alkaloids and related derivatives is described. Our synthesis is predicated on the convergent and modular diazene-directed assembly of two complex fragments to secure the critical $\mathrm{C} 3 \mathrm{a}-\mathrm{C} 3 \mathrm{a}^{\prime}$ linkage followed by a guided biomimetic aminal reorganization to deliver the heptacyclic core of these alkaloids. Concise enantioselective syntheses of the fragments were devised, with highlights including the application of a rationally designed sulfinamide chiral auxiliary,
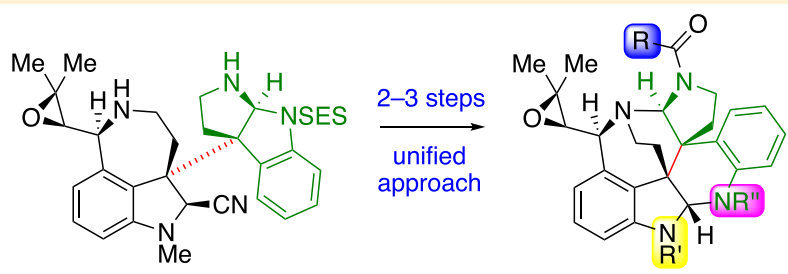

- synthesis of all epoxy communesins - evaluation against five carcinomas - revision of $(-)$-communesin I, (C3"R) - potent new anticancer derivatives an efficient calcium trifluoromethanesulfonate promoted intramolecular amination, and a diastereoselective epoxidation that simultaneously converts the new chiral auxiliary to a versatile amine protective group. The modularity of our convergent approach enabled the rapid synthesis of all epoxidecontaining members of the communesin family from a single heterodimeric intermediate, including the first total synthesis of communesins $\mathrm{C}-\mathrm{E}$, and $\mathrm{G}-\mathrm{I}$, and facilitated our stereochemical revision of (-)-communesin I, the most recently isolated communesin alkaloid. Furthermore, the generality of our biogenetically inspired heterodimer rearrangement was demonstrated in a guided synthesis of a communesin derivative with an unnatural topology. Finally, we report the first comparative analysis of the anticancer activities of all naturally occurring communesin alkaloids A-I and eight complex derivatives against five human cancer cell lines. From these data, we have identified (-)-communesin B as the most potent natural communesin and discovered that derivatives with $\mathrm{N8}^{\prime}$-sulfonamide substitution exhibit up to a 10 -fold increase in potency over the natural alkaloids.

\section{INTRODUCTION}

The communesin alkaloids are a family of nine polycyclic natural products, which possess notable biological activities and a range of interesting structural features (Figure 1$){ }^{1}$ (-)-Communesin A (2) and B (4) were first isolated in 1993 by Numata and co-workers from an algal-derived Penicillium fungus and were found to exhibit moderate to potent cytotoxicity against cultured murine lymphocytic leukemia cells. $^{1 \mathrm{a}}$ In the years since, seven more communesin alkaloids $\mathrm{C}-\mathrm{I}$ (Figure 1) have been isolated and shown to possess insecticidal, antiproliferative, and vasculogenetic activities. ${ }^{1}$ The core structures of these alkaloids feature seven contiguous rings, two aminal linkages, and up to six stereogenic centers, of which two are vicinal and quaternary (C3a/C3a'). This formidable structural complexity coupled with an array of important biological activity prompted research efforts directed toward their total chemical synthesis, culminating in inventive solutions for the preparation of racemic ${ }^{2}$ and enantiomerically enriched $^{3}$ samples of communesin F (1). However, access to the more complex C10-epoxide-containing communesins remains challenging. Indeed, other than Zuo and Ma's total synthesis of (-)-communesin A (2) and B (4) in 2011, ${ }^{4}$ there have been no reports describing the synthesis of epoxycommunesins 2-9. Therefore, we sought to develop a unified and convergent approach to all members of the communesin family and related complex derivatives to enable their detailed study. Herein, we report the first biomimetic enantioselective total synthesis of all eight known epoxide-bearing communesin alkaloids, including the first total synthesis of communesins $\mathrm{C}-\mathrm{E}$, and $\mathrm{G}-\mathrm{I}$, a set of unnatural analogues, and their side-byside anticancer profiling against five human cancer cell lines. In addition, we revise the reported structure of $(-)$-communesin I $(9)^{1 \mathrm{f}}$ to $(-)-\left(3^{\prime \prime} R\right)$-communesin I (10, Figure 1$)$.

In 2016, we reported the first biomimetic enantioselective total synthesis of (-)-communesin F (1) inspired by a hypothetical biosynthesis involving the oxidative union of Penicillium fungal alkaloid (-)-aurantioclavine (11) and tryptamine $(12$, Scheme 1$) .{ }^{3 \mathrm{~b}}$ We anticipated that the resulting $\mathrm{C} 3 \mathrm{a}-\mathrm{C} 3 \mathrm{a}^{\prime}$ linked heterodimer $\mathbf{1 5}$ would undergo a dynamic reorganization to afford the key structural elements of the communesin core 16. Fortuitously, Tang, Garg, and Houk

Received: July 11, 2019

Published: August 17, 2019 


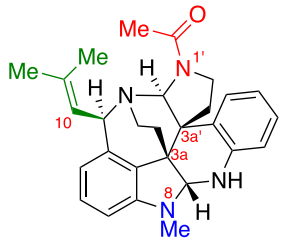

(-)-communesin F (1)

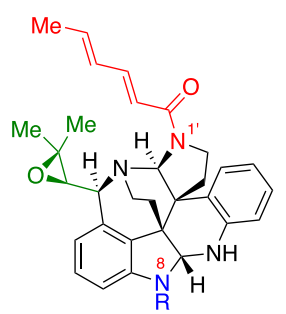

(-)-communesin $\mathrm{B}(4), \mathrm{R}=\mathrm{Me}$ $(-)$-communesin $\mathrm{C}(5), \mathrm{R}=\mathrm{H}$ (+)-communesin D (6), $\mathrm{R}=\mathrm{CHO}$

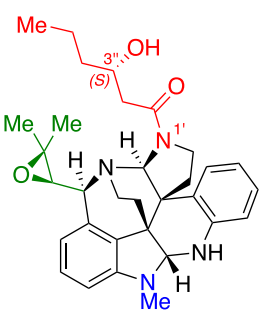

(-)-(3"S)-communesin I (9) reported stereochemistry (ref. 1f)

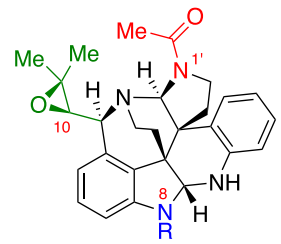

(-)-communesin $\mathrm{A}(2), \mathrm{R}=\mathrm{Me}$ $(-)$-communesin $\mathrm{E}(3), \mathrm{R}=\mathrm{H}$

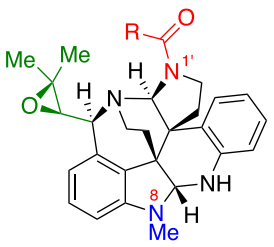

(-)-communesin $\mathrm{G}(7), \mathrm{R}=\mathrm{Et}$ $(-)$-communesin $\mathrm{H}(\mathbf{8}), \mathrm{R}=n-\mathrm{Pr}$

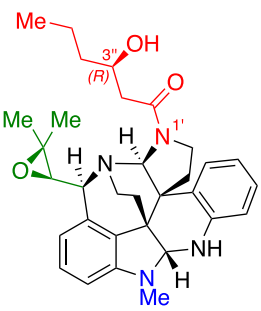

$(-)-(3 " R)$-communesin I (10) revised stereochemistry (this report)

Figure 1. Structures of the Communesin Alkaloids.

Scheme 1. Biogenesis of the Communesin Alkaloids

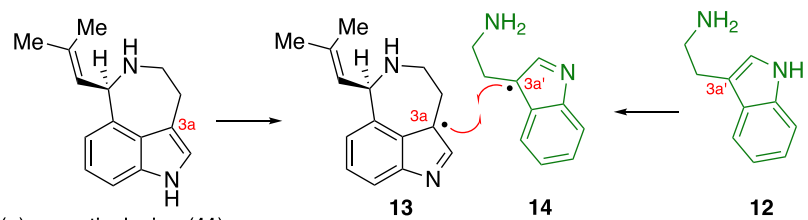

(-)-aurantioclavine (11)

13

12

$\downarrow$ radical coupling

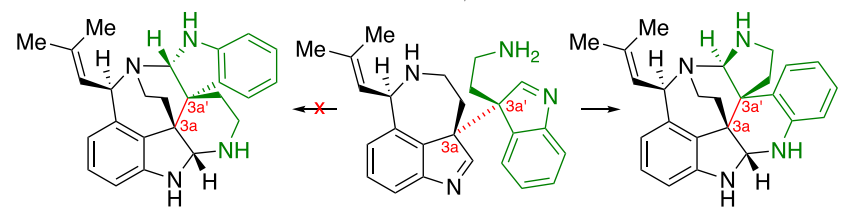

iso-communesin core 17

15

communesin core 16

disclosed landmark biosynthetic and computational studies ${ }^{5}$ which were in full accord with our biosynthetic hypotheses. They demonstrated that alkaloids (-)-11 and 12 were essential biosynthetic precursors and, further, they identified a Penicillium P450 monooxygenase enzyme (CnsC) responsible for both their oxidative union and selective conversion to heptacycle 16 in preference to isomeric heptacycle 17.

Our biomimetic approach to alkaloid (-)-1 was predicated on the late-stage diazene-directed assembly of two amine fragments to secure the key $\mathrm{C} 3 \mathrm{a}-\mathrm{C} 3 \mathrm{a}^{\prime}$ linkage with complete stereochemical control. ${ }^{3 \mathrm{~b}} \mathrm{We}$ then deployed the resulting heterodimeric structure as a surrogate for the hypothetical biosynthetic intermediate 15 , which enabled the examination of conditions for a guided biomimetic aminal reorganization to furnish the core of the communesin alkaloids to the exclusion

of other possible constitutional isomers. From this penultimate intermediate, we were able to access (-)-communesin F (1) in only one additional step. The success of our strategy to alkaloid (-)-1 prompted the development of a unified approach to access the challenging epoxy-communesin alkaloids 2-10 (Figure 1) via a convergent synthesis while accommodating acid sensitive intermediates bearing the $\mathrm{C} 10$ epoxide.

\section{RESULTS AND DISCUSSION}

As depicted in our representative retrosynthetic analysis of (-)-communesin A (2, Scheme 2), we envisioned access to all

Scheme 2. Retrosynthetic Analysis of (-)-Communesin A

(2)

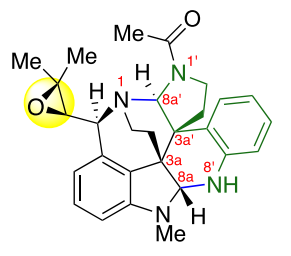

$(-)$-communesin A (2)

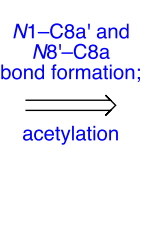
formation

etylation

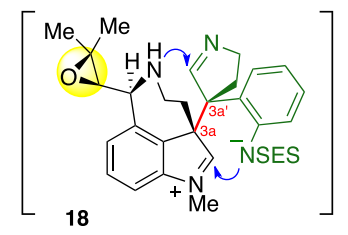

18

$\| \begin{array}{r}\text { biomimetic } \\ \text { reorganization }\end{array}$
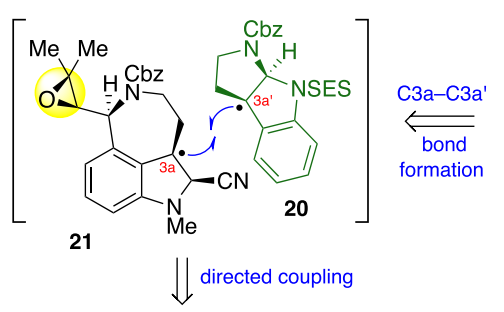

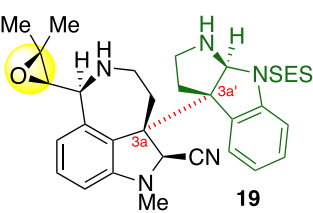

key intermediate

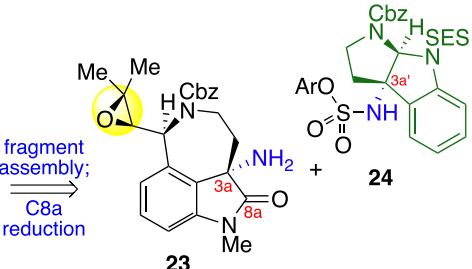

C10-epoxy-communesins 2-9 via a late-stage biomimetic aminal reorganization of the intermediate epoxy-heterodimer 19 followed by $\mathrm{N} 1^{\prime}$ acylation. Importantly, we anticipate this late-stage $\mathrm{N1}^{\prime}$-acylation will enable access to all members of this alkaloid class containing diverse $\mathrm{N} 1^{\prime}$ substituents. Next, consistent with our diazene-directed strategy for complex fragment assembly, ${ }^{3 b, 6}$ we projected that the critical C3a-C $3 a^{\prime}$ linkage in 19 could be assembled via photoextrustion of dinitrogen from unsymmetrical diazene 22 and recombination of the resulting radical fragments 20 and $\mathbf{2 1}$. We expected to assemble diazene $\mathbf{2 2}$ from epoxide-bearing C3a-amino oxindole 23 and $\mathrm{C} 3 \mathrm{a}^{\prime}$-sulfamate 24 . We envisioned an early stage epoxidation to permit the key biomimetic rearrangement and to fully exploit our versatile and convergent approach. This strategy provides maximally divergent access to all C10-epoxycommunesins 2-9 from a single advanced synthetic intermediate, heterodimer 19.

Our total synthesis of alkaloids $\mathbf{2 - 1 0}$ (Figure 1) began with the preparation of oxindole 23 and sulfamate 24 , the key amine fragments required for the synthesis of complex diazene 22 . The application of our silver(I)-mediated substitution chemistry enabled rapid and scalable access to sulfamate $(+)-24$ (Scheme 3). Electrophilic activation of readily available enantioenriched $\mathrm{C} 3 \mathrm{a}^{\prime}$-bromocyclotryptamine $(+)-25^{3 \mathrm{~b}, 6 \mathrm{~d}, 7}$ 
Scheme 3. Gram-Scale Synthesis of Sulfamate (+)-24

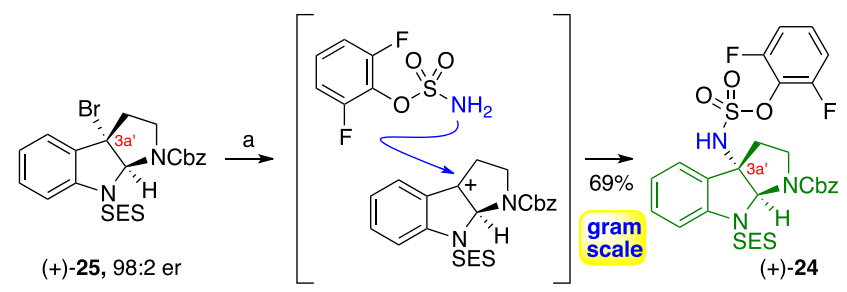

${ }^{a}$ Reagents and conditions: (a) AgOTf, 2,6-di-tert-butyl-4-methylpyridine, 2,6-difluorophenyl sulfamate, $\mathrm{CH}_{2} \mathrm{Cl}_{2}, 23{ }^{\circ} \mathrm{C}$.

with silver(I) trifluoromethanesulfonate in the presence of 2,6difluorophenylsulfamate and 2,6-di-tert-butyl-4-methylpyridine (DTBMP) afforded the corresponding sulfamate ester (+)-24 in $69 \%$ yield.

Having secured an efficient gram-scale synthesis of cyclotryptamine $(+)-\mathbf{2 4}$, we turned our attention to the preparation of amino azepane fragment $\mathbf{2 3}$ which contains the key (C10R)configured epoxide, a critical structural feature found in communesins 2-10. Our initial efforts directed toward the synthesis of this intermediate and related derivatives revealed a pronounced acid-sensitivity of the C10-epoxide, which stems from facile intramolecular opening of the protonated epoxide with the N1-carbamate to form stable oxazolidinone products. ${ }^{8}$ This precluded the use of Ellman's tert-butanesulfinamide chiral auxiliary, ${ }^{9}$ which we previously employed en route to (-)-communesin F (1)..$^{3 \mathrm{~b}}$ Specifically, epoxidation of intermediates containing Ellman's auxiliary (i.e., the tert-butanesulfinamide variant of 32) resulted in rapid concomitant oxidation of the sulfinamide to the corresponding tertbutanesulfonamide (Bus), which requires strong Bronsted acids, such as trifluoromethanesulfonic acid, to remove. ${ }^{10}$ This unforeseen incompatibility prompted our design of 2(trimethylsilyl)ethane sulfinamide (26), a new sulfinamide auxiliary whose oxidation product, 2-(trimethylsilyl)ethane sulfonamide (SES), can be removed under nonacidic and nonreducing conditions, ${ }^{11}$ an essential requirement for the preservation of the sensitive C10-epoxide (Scheme 4).

Multigram quantities of enantiopure $(S)$-sulfinamide (-)-26 were prepared using readily available $(-)$-diacetone-Dglucose $^{12}$ as a chiral controller. ${ }^{13}$ Condensation of the new chiral auxiliary (-)-26 with $\mathrm{N}$-methyl-4-bromoisatin in the presence of titanium(IV) ethoxide ${ }^{3 \mathrm{~b}, 9}$ then afforded the corresponding sulfinyl imine (+)-27 in $80 \%$ yield. Subsequent allylation with allylmagnesium bromide afforded the corresponding addition product (+)-28 in $74 \%$ yield as a single diastereomer on a multigram scale after flash column chromatography. We were pleased to discover that the inherent diastereoselectivity imparted by this new auxiliary (84:16 dr) was remarkably similar to that observed with Ellman's tert-butanesulfinamide $(87: 13 \mathrm{dr})$ under identical reaction conditions, ${ }^{3 \mathrm{~b}, 9}$ thereby validating the broader utility of sulfinamide $(-)-26$ in stereoselective synthesis.

Ozonolysis of alkene (+)-28 followed by in situ ozonide reduction with sodium borohydride furnished primary alcohol (+)-29 in $85 \%$ yield. Mitsunobu displacement of the alcohol with $\mathrm{N}$-carbobenzoxy-2-nitrobenzenesulfonamide (o$\mathrm{NsNHCbz}$ ) and in situ desulfonylation then afforded benzyl carbamate (+)-30 in $76 \%$ overall yield. A palladium-catalyzed Mizoroki-Heck reaction with 1,1-dimethylallyl alcohol and silver(I) carbonate as the base then proceeded to furnish allylic
Scheme 4. Synthesis of Epoxide (-)-33 and Determination of Relative Stereochemistry at $\mathrm{C}^{a}{ }^{a}$<smiles>C=CCC1(NS(=O)CC[As])C(=O)Nc2cccc(Br)c2C1([14CH3])Br</smiles>

$(+)-27$

(+)-28

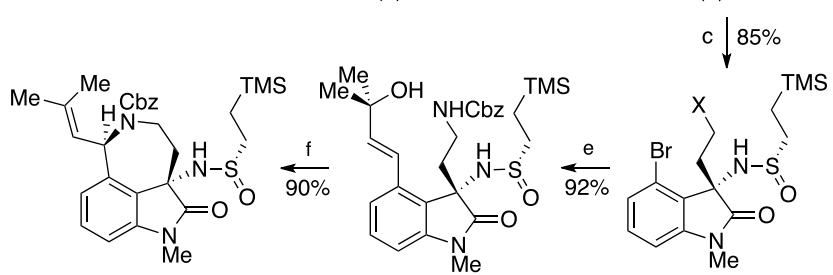

$(-)-32$

$(-)-31$

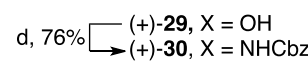

g $\downarrow$ gram scale

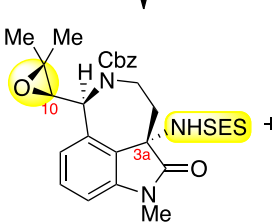

$(-)-33,81 \%$

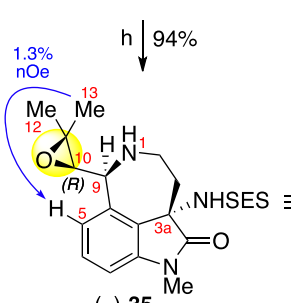

$(-)-35$

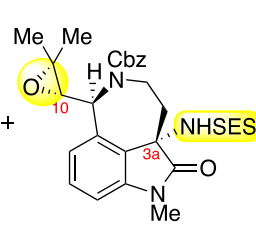

$(-)-34,8 \%$

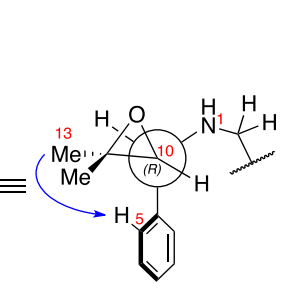

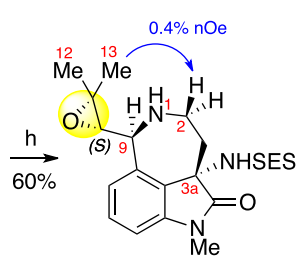

$(-)-36, X$-ray

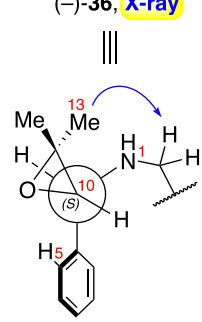

${ }^{a}$ Reagents and conditions: (a) $\mathrm{N}$-methyl-4-bromoisatin, $\mathrm{Ti}(\mathrm{OEt})_{4}$, $\mathrm{CH}_{2} \mathrm{Cl}_{2}, 23{ }^{\circ} \mathrm{C}$; (b) allylMgBr, $\mathrm{MgBr}_{2}, \mathrm{CH}_{2} \mathrm{Cl}_{2},-78{ }^{\circ} \mathrm{C}$, > 99:1 dr; (c) $\mathrm{O}_{3}, \mathrm{MeOH},-78{ }^{\circ} \mathrm{C} ; \mathrm{NaBH}_{4},-78{ }^{\circ} \mathrm{C} \rightarrow 23{ }^{\circ} \mathrm{C}$; (d) o-NsNHCbz, DIAD, $\mathrm{PPh}_{3}, \mathrm{THF}, 50{ }^{\circ} \mathrm{C}$; $\mathrm{PhSH}, \mathrm{Cs}_{2} \mathrm{CO}_{3}, 50{ }^{\circ} \mathrm{C}$; (e) $1,1-$ dimethylallyl alcohol, $\mathrm{Pd}(\mathrm{OAc})_{2}, \mathrm{Ag}_{2} \mathrm{CO}_{3}, \mathrm{DMF}-\mathrm{H}_{2} \mathrm{O}, 90{ }^{\circ} \mathrm{C}$; (f) $\mathrm{Ca}(\mathrm{OTf})_{2}, \mathrm{MeCN}, 80{ }^{\circ} \mathrm{C}$; (g) 1,1,1-trifluoroacetone, $30 \%$ aq. $\mathrm{H}_{2} \mathrm{O}_{2}$, $\mathrm{K}_{2} \mathrm{CO}_{3}$, EDTA, $\mathrm{MeCN}-\mathrm{H}_{2} \mathrm{O}, 0{ }^{\circ} \mathrm{C}$; (h) $\mathrm{H}_{2}, \mathrm{Pd}(\mathrm{OH})_{2} / \mathrm{C}$, EtOH, 23 ${ }^{\circ} \mathrm{C}$.

alcohol (-)-31 in 92\% yield. ${ }^{14}$ Unexpectedly, subjecting (-)-31 to our previously employed palladium-catalyzed allylic amination conditions $\left(\mathrm{PdCl}_{2} \mathrm{MeCN}_{2}, \mathrm{MeCN}, 80{ }^{\circ} \mathrm{C}\right)^{3 \mathrm{~b}, 15}$ resulted in complex mixtures containing only trace amounts of azepane (-)-32. The major side products were derived from sulfinamide epimerization and desulfinylation. We hypothesize that the transiently generated hydrochloric acid necessary for catalyst turnover ${ }^{16}$ resulted in sulfinamide cleavage and release of the free amine and the corresponding sulfinyl chloride, which is expected to be configurationally unstable. ${ }^{17}$ Recombination of the amine and the racemized sulfinyl chloride would then afford the observed diastereomeric sulfinamide. After extensive experimentation, we discovered that calcium(II) trifluoromethanesulfonate and related Lewis acids $^{18}$ could promote a highly efficient allylic amination without concomitant sulfinamide degradation. Indeed, under optimal conditions, gram scale synthesis of azepane (-)-32 was achieved in $90 \%$ yield. 
We then focused on the introduction of the critical C10epoxide. We were pleased to discover that mild, efficient, and stereoselective epoxidation of this key intermediate could be achieved using in situ generated methyl(trifluoromethyl)dioxirane (TFDO). ${ }^{19,20}$ Exposure of an acetonitrile solution of (-)-32 to aqueous potassium carbonate and aqueous hydrogen peroxide in the presence of 1,1,1-trifluoroacetone at $0{ }^{\circ} \mathrm{C}$ furnished the desired (C10R)-configured epoxide (-)-33 in $81 \%$ yield in addition to the $(\mathrm{C} 10 S)$-configured epoxide (-)-34 in $8 \%$ yield, coupled with concomitant and planned oxidation of the alkane sulfinamide to the corresponding 2(trimethylsilyl)ethane sulfonamide (SES). ${ }^{21}$

The relative configuration at $\mathrm{C} 10$ of these epimeric epoxides was determined by nuclear Overhauser effect analysis on free amines (-)-35 and (-)-36 after hydrogenolytic removal of the benzyl carbamates (Scheme 4). According to Murata's $J_{\mathrm{H}-\mathrm{H}^{-}}$ based method ${ }^{22}$ as employed by Proksch, ${ }^{\text {,b }}$ Christophersen, ${ }^{\text {le }}$ and Chen ${ }^{1 \mathrm{f}}$ for communesins 4-9, the large coupling constant between $\mathrm{C} 9 \mathrm{H}$ and $\mathrm{C} 10 \mathrm{H}(J \approx 9.0 \mathrm{~Hz})$ in both epoxides (-)-35 and (-)-36 indicates an approximately $180^{\circ}$ dihedral angle between $\mathrm{C} 9 \mathrm{H}$ and $\mathrm{C} 10 \mathrm{H}$, affording two possible diastereomeric anti configurations. In the case of epoxide (-)-35, the NOE enhancement observed at $\mathrm{C} 5 \mathrm{H}$ when irradiating the geminal methyl groups is consistent with a syn orientation between the epoxide oxygen and N1 as depicted in the Newman projection in Scheme 4 and the $(R)$-configuration at $\mathrm{C} 10$. Conversely, the NOE enhancements observed at $\mathrm{C} 2 \mathrm{H}$ in epoxide (-)-36 when irradiating the geminal methyl groups suggests an anti orientation between the epoxide oxygen and $\mathrm{N} 1$ and thus the $(S)$-configuration at C10. Indeed, this assignment of epoxide (-)-36 was later unambiguously confirmed by single-crystal X-ray diffraction. ${ }^{13}$

With a stereoselective synthesis of epoxide (-)-33 in hand, we moved to investigate $\mathrm{C} 8 \mathrm{a}$ reduction and unveiling of the $\mathrm{C} 3 \mathrm{a}$ amine as a prelude to our planned diazene synthesis. We initially targeted the introduction of a C8a-nitrile, which we have shown to be an ideal trigger for late-stage C8a-iminiun ion formation while providing adequate stability during the fragment assembly steps (Scheme 5). ${ }^{3 \mathrm{~b}}$ To this end, partial

Scheme 5. Synthesis of Amine Fragments (+)-38 and $(-)-23^{a}$
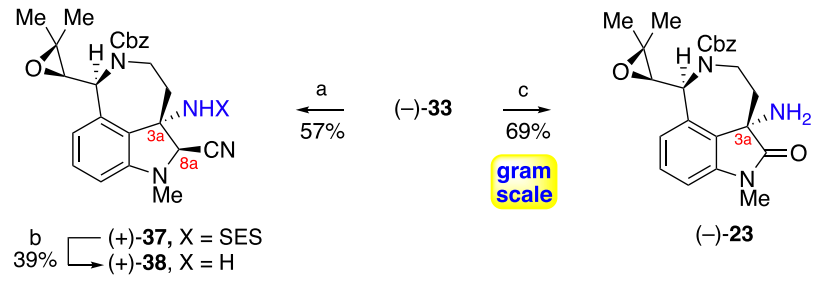

${ }^{a}$ Reagents and conditions: (a) (i) $\mathrm{LiBH}_{4}, \mathrm{MeOH}, \mathrm{THF}, 23{ }^{\circ} \mathrm{C}$; (ii) TMSCN, $\mathrm{H}_{2} \mathrm{O}$, HFIP, $0{ }^{\circ} \mathrm{C} \rightarrow 23{ }^{\circ} \mathrm{C}$. (b) TASF, DMF, $100{ }^{\circ} \mathrm{C}$. (c) TASF, $\mathrm{H}_{2} \mathrm{O}$, DMF, $100{ }^{\circ} \mathrm{C}$.

reduction of oxindole (-)-33 with lithium borohydride afforded the corresponding C8a-hemiaminal as a mixture of diastereomers. Treatment of the crude hemiaminal with trimethylsilyl cyanide in wet hexafluoroisopropanol (HFIP) afforded aminonitrile (+)-37 in 57\% yield. ${ }^{13,23}$ Fluoridemediated $\mathrm{C} 3 \mathrm{a}-\mathrm{N}$ desulfonylation with tris(dimethylamino)sulfonium difluorotrimethylsilicate (TASF) $)^{24}$ in anhydrous $\mathrm{N}, \mathrm{N}$-dimethylformamide (DMF) at $100{ }^{\circ} \mathrm{C}$ provided benzylic amine (+)-38 in 39\% yield, with the C8a-epimer and the C8acyanoindole elimination product comprising the remainder of the mass balance. Attempts to attenuate the basicity of the reagent by the addition of water or other acidic additives proved unsuccessful in reducing the propensity of the substrate to undergo elimination or epimerization. To circumvent problematic side reactions, we opted to desulfonylate oxindole (-)-33 directly and investigate C8a reduction after the fragment assembly. Indeed, treatment of sulfonamide (-)-33 with TASF in wet DMF at $100{ }^{\circ} \mathrm{C}$ provided gram-scale access to amino-oxindole (-)-23 in $69 \%$ yield.

With both critical amine fragments in hand, we then moved to explore their union and the introduction of the key C3aC3a' linkage. Simply stirring a tetrahydrofuran solution of amine (-)-23 and sulfamate (+)-24 in the presence of $4-(N, N$ dimethylamino)pyridine (DMAP) afforded oxindole sulfamide (-)-39 in $84 \%$ yield on a gram scale (Scheme 6). Partial

Scheme 6. Directed Synthesis of Heterodimer (+)-19 via Diazene-Directed Fragment Assembly ${ }^{a}$

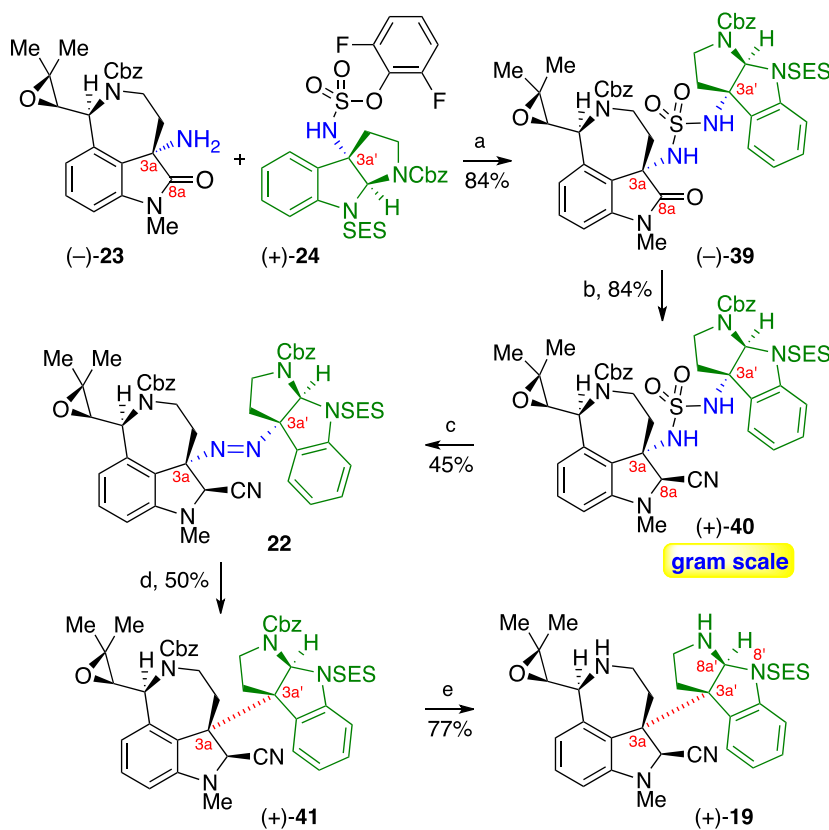

${ }^{a}$ Reagents and conditions: (a) DMAP, THF, $23{ }^{\circ} \mathrm{C}$. (b) (i) $\mathrm{LiBH}_{4}$ $\mathrm{MeOH}, \mathrm{THF}, 23{ }^{\circ} \mathrm{C}$; (ii) TMSCN, $\mathrm{H}_{2} \mathrm{O}$, HFIP, $0 \rightarrow 23{ }^{\circ} \mathrm{C}$. (c) polystyrene-2-tert-butylimino-2-diethylamino-1,3-dimethylperhydro-1,3,2-diazaphosphorine, $N$-chloro- $N$-methylbenzamide, $\mathrm{MeOH}$, $23{ }^{\circ} \mathrm{C}$. (d) $h \nu(350 \mathrm{~nm}), 25{ }^{\circ} \mathrm{C}$. (e) $\mathrm{H}_{2}, \mathrm{Pd}(\mathrm{OH})_{2} / \mathrm{C}, \mathrm{EtOH}, 23{ }^{\circ} \mathrm{C}$.

reduction of the oxindole with excess lithium borohydride and treatment of the resulting hemiaminal with trimethylsilyl cyanide in wet $^{25}$ hexafluoroisopropanol then afforded aminonitrile sulfamide $(+)-\mathbf{4 0}$ as a single diastereomer in $84 \%$ overall yield on a gram scale. Fortuitously, formation of the C8a-nitrile after fragment assembly proved to be much more efficient and diastereoselective, likely due to the steric bulk of the cyclotryptamine moiety that more effectively shields the bottom face of the C8a-iminium.

Exposure of $(+)-40$ to $N$-chloro- $N$-methylbenzamide in the presence of polystyrene-bound 2-tert-butylimino-2-diethylamino-1,3-dimethylperhydro-1,3,2-diazaphosphorine (BEMP) in methanol then afforded sensitive diazene $\mathbf{2 2}$ in $45 \%$ yield, without competitive oxidation of the electron-rich arene. ${ }^{3 \mathrm{~b}}$ 
Scheme 7. Synthesis of All Known Epoxide-Bearing Communesins and Stereochemical Revision of (-)-Communesin I (10) ${ }^{a}$

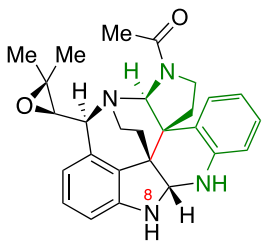

(-)-communesin $\mathrm{E}(3)$

i $\uparrow 81 \%$

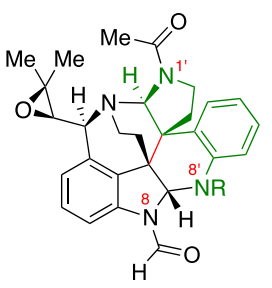

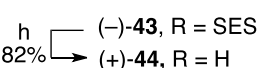

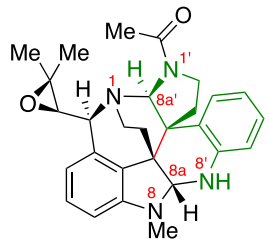

(-)-communesin A (2)

h $\uparrow 77 \%$

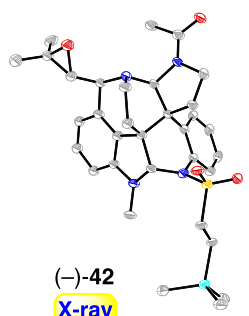

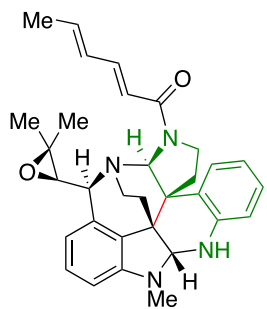

(-)-communesin B (4)

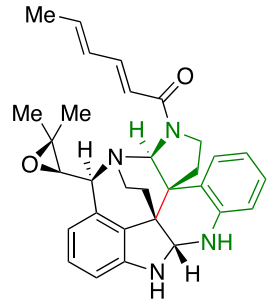

(-)-communesin C (5)

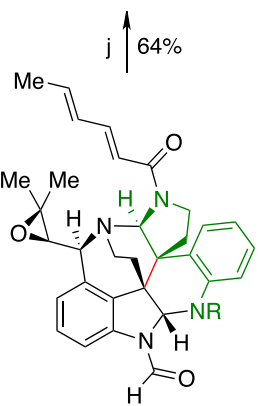

+ )-46, R = SES

(+)-communesin D (6), $\mathrm{R}=\mathrm{H}$
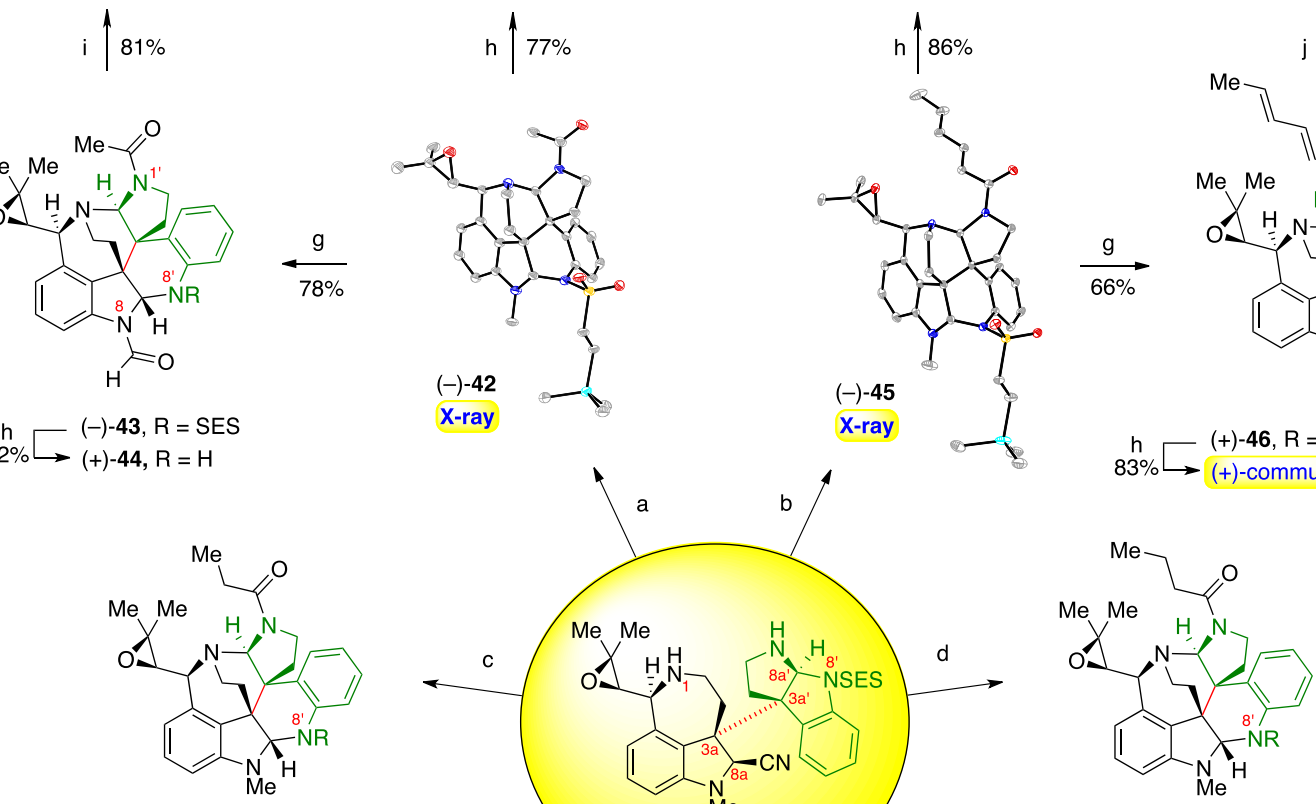

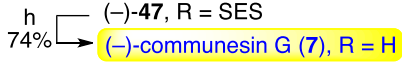

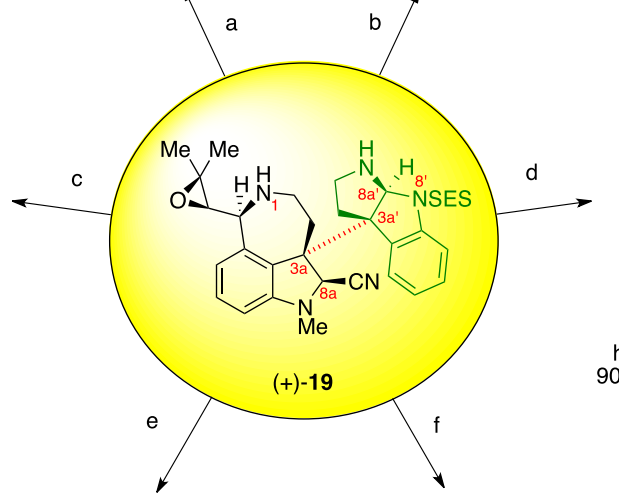

h $-(-)-48, R=S E S$

$90 \% \square(-)$-communesin $\mathrm{H}(8), \mathrm{R}=\mathrm{H}$

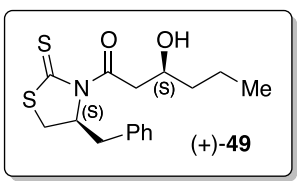

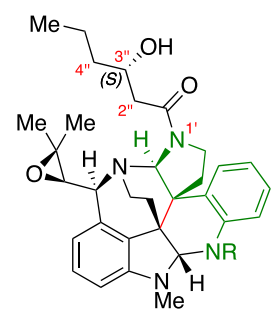

h $-(-)-50, R=S E S$

$86 \% \square(-)-(3 " S)$-communesin I (9) $\mathrm{R}=\mathrm{H}$ Reported stereochemistry (ref. 1f)

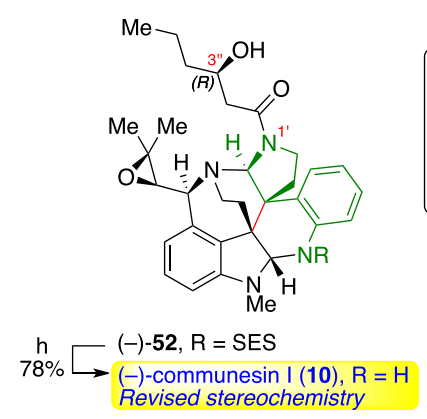

${ }^{a}$ Reagents and Conditions: (a) $t$-BuOLi, EtOH $60{ }^{\circ} \mathrm{C}$; PPTS, Ac 2 O, $23{ }^{\circ} \mathrm{C}, 82 \%$. (b) $t$-BuOLi, EtOH, $60{ }^{\circ} \mathrm{C}$; PPTS, sorbic anhydride, $23{ }^{\circ} \mathrm{C}$, $82 \%$. (c) $t$-BuOLi, EtOH, $60{ }^{\circ} \mathrm{C}$; PPTS, propionic anhydride, $23{ }^{\circ} \mathrm{C}, 86 \%$ (d) $t$-BuOLi, EtOH, $60{ }^{\circ} \mathrm{C}$; PPTS, butyric anhydride, $23{ }^{\circ} \mathrm{C}$, $84 \%$. (e) $t$ BuOLi, EtOH, $60{ }^{\circ} \mathrm{C}$; PPTS, (+)-49, $23{ }^{\circ} \mathrm{C}$, 84\%. (f) t-BuOLi, EtOH, $60{ }^{\circ} \mathrm{C}$; PPTS, (+)-51, $23{ }^{\circ} \mathrm{C}$, 48\%. (g) pyridinium dichromate (PDC), $\mathrm{K}_{2} \mathrm{CO}_{3}, 1,2$-dichloroethane, $60{ }^{\circ} \mathrm{C}$. (h) TASF, DMF, $23{ }^{\circ} \mathrm{C}$. (i) (i) $\mathrm{KOH}, \mathrm{H}_{2} \mathrm{O}-\mathrm{DMSO}, 23{ }^{\circ} \mathrm{C}$; (ii) $\mathrm{TASF}, \mathrm{DMF}, 45{ }^{\circ} \mathrm{C}$. (j) (i) KOH, $\mathrm{H}_{2} \mathrm{O}-$ DMSO, $23{ }^{\circ} \mathrm{C}$; (ii) TASF, DMF, $23^{\circ} \mathrm{C}$. In the ORTEP representations of sulfonamide $(-)-42$ and $(-)-45$, the thermal ellipsoids are drawn at $30 \%$ probability.

Photoexcitation and expulsion of dinitrogen from a thin film of diazene 22 afforded the $\mathrm{C} 3 \mathrm{a}-\mathrm{C} 3 \mathrm{a}^{\prime}$ linked heterodimer $(+)-41$ in $50 \%$ yield as a single diastereomer via a completely stereoselective combination of the radical fragments $\mathbf{2 0}$ and $\mathbf{2 1}$ (Scheme 2). ${ }^{26}$

Hydrogenolysis of the benzyl carbamates then furnished heterodimeric diamine (+)-19 in 77\% yield, setting the stage for our key biomimetic aminal reorganization. Consistent with the design principles underpinning our synthetic strategy, the position of the electron withdrawing group on the cyclotryptamine moiety enables selective cleavage of either aminal linkage, thereby controlling the regiochemical outcome of the rearrangement (vide infra). The $\mathrm{N}^{\prime}$-sulfonamide of diamine (+)-19 was expected to guide the cleavage of the $\mathrm{C} 8 \mathrm{a}^{\prime}-\mathrm{N} 8{ }^{\prime}$ bond under basic conditions, leading to the heptacyclic core of 
the communesin alkaloids after formation of the $\mathrm{C} 8 \mathrm{a}-\mathrm{N} 8^{\prime}$ and C8 $\mathrm{a}^{\prime}-\mathrm{N} 1$ aminals (Scheme 2).

With the critical intermediate heterodimer (+)-19 in hand, we turned our attention to the preparation of all known epoxide-containing members of the communesin family, beginning with $N 1^{\prime}$-acetyl communesins (-)-2 and (-)-3, respectively. Importantly, we observed that a clean and complete rearrangement to the epoxide-appended communesin core could be achieved by exposing heterodimer (+)-19 to ethanolic lithium tert-butoxide at $60{ }^{\circ} \mathrm{C}$ (Scheme 7$) .{ }^{27}$ In situ neutralization of excess alkoxide with pyridinium $p$-toluenesulfonate (PPTS) followed by acetylation of the resulting sensitive heptacycle with acetic anhydride then furnished communesin derivative (-)-42 in $82 \%$ yield. Analysis of the advanced intermediate (-)-42 by single-crystal X-ray diffraction unambiguously confirms the stereochemical configuration of the C10-epoxide and provided the first solid-state structure of the full polycyclic topology of the communesin alkaloids. ${ }^{13}$ The structure of intermediate (-)-42 illustrates the compressed pyramidal N1-amine, ${ }^{28}$ the preferred orientation of the $\mathrm{N} 1^{\prime}$-acetyl group, and the positioning of the $\mathrm{N} 8$ ' sulfonyl group, a substitution critical to the enhanced anticancer activity observed of new communesin derivatives (vide infra). Treatment of (-)-N8'-SES-communesin A (42) with TASF in degassed ${ }^{29}$ DMF provided (-)-communesin A (2) in $77 \%$ yield. All ${ }^{1} \mathrm{H}$ and ${ }^{13} \mathrm{C}$ NMR data as well as optical rotation (observed $[\alpha]_{\mathrm{D}}{ }^{24}=-165\left(c=0.39, \mathrm{CHCl}_{3}\right)$; lit: $[\alpha]_{\mathrm{D}}{ }^{22}=-58\left(c=0.14, \mathrm{CHCl}_{3}\right),{ }^{1 \mathrm{a}}[\alpha]_{\mathrm{D}}{ }^{20}=-174(c=1.34$, $\left.\left.\mathrm{CHCl}_{3}\right),{ }^{1 \mathrm{c}}[\alpha]_{\mathrm{D}}{ }^{30}=-163.5\left(c=0.14, \mathrm{CHCl}_{3}\right)^{4}\right)$ for synthetic alkaloid (-)-2 were consistent with literature values.

Oxidation of (-)-N8'-SES-communesin A (42) with pyridinium dichromate (PDC, 10 equiv) ${ }^{2 \mathrm{~d}, 30}$ and potassium carbonate (40 equiv) in 1,2-dichloroethane at $60{ }^{\circ} \mathrm{C}$ provided the corresponding N8-formyl derivative (-)-43 in $78 \%$ yield. Removal of the $\mathrm{N} 8^{\prime}$-sulfonyl group from intermediate $(-)-43$ resulted in (+)-N8-formyl communesin E (44) in $82 \%$ yield. Interestingly, this communesin derivative has not yet been isolated in nature to date, which is notable given that natural samples of $(+)$-communesin $\mathrm{D}(6)$, the closely related $N 1^{\prime}$ sorbyl derivative, have been repeatedly and independently isolated. ${ }^{1 \mathrm{~b}, \mathrm{c}}$ Alternatively, mild hydrolysis of formamide (-)-43 with potassium hydroxide in wet dimethyl sulfoxide (DMSO) followed by desulfonylation afforded the first synthesis of $(-)$-communesin E (3) in $81 \%$ yield. All spectral data and optical rotation (observed $[\alpha]_{\mathrm{D}}^{23}=-191\left(c=0.31, \mathrm{CHCl}_{3}\right)$; lit: $\left.[\alpha]_{\mathrm{D}}{ }^{20}=-156\left(c=0.11, \mathrm{CHCl}_{3}\right)^{1 \mathrm{c}}\right)$ for alkaloid $(-)-3$ were in agreement with the isolation report.

We next focused on the synthesis of $\mathrm{N}^{\prime}$ '-sorbyl alkaloids (-)-communesins B-D (4-6, respectively). Treatment of heterodimeric diamine $(+)-19$ with the aminal rearrangement conditions described above followed by acylation with sorbic anhydride afforded (-)-N8'-SES-communesin B (45) in $82 \%$ yield. Mild N8'-desulfonylation with TASF then afforded (-)-communesin B (4) in $86 \%$ yield, whose spectroscopic data as well as optical rotation (observed $[\alpha]_{\mathrm{D}}^{23}=-64(c=0.46$, $\left.\mathrm{CHCl}_{3}\right)$; lit: $[\alpha]_{\mathrm{D}}{ }^{22}=+8.7\left(c=0.23, \mathrm{CHCl}_{3}\right),{ }^{1 \mathrm{a}}[\alpha]_{\mathrm{D}}=-58(c$ $=0.10, \mathrm{MeOH}),{ }^{1 \mathrm{~b}}[\alpha]_{\mathrm{D}}{ }^{20}=-74.9\left(c=1.50, \mathrm{CHCl}_{3}\right),{ }^{1 \mathrm{c}}[\alpha]_{\mathrm{D}}{ }^{30}$ $\left.=-51.3\left(c=0.30, \mathrm{CHCl}_{3}\right)^{4}\right)$ were consistent with previously reported values, with the exception of the anomalous positive value described in the first isolation report. ${ }^{1 \mathrm{a}, 31}$ Oxidation of advanced intermediate (-)-45 with PDC provided the sensitive $N 8$-formamide (+)-46 in $66 \%$ yield which was then desulfonylated to provide the first synthesis of $(+)$-commune- sin $\mathrm{D}(6)$ in $83 \%$ yield. All ${ }^{1} \mathrm{H}$ and ${ }^{13} \mathrm{C}$ data as well as optical rotation (observed $[\alpha]_{\mathrm{D}}{ }^{23}=+151\left(c=0.23, \mathrm{CHCl}_{3}\right)$; lit: $\left.[\alpha]_{\mathrm{D}}{ }^{20}=+150\left(c=0.14, \mathrm{CHCl}_{3}\right)^{1 \mathrm{c}}\right)$ of $(+)-6$ were fully consistent with literature values. To complete the synthesis of all known $\mathrm{N} 1$ '-sorbyl communesin derivatives, deformylation of the N8-formamide (+)-46 followed by desulfonylation of the resulting crude amine produced (-)-communesin $\mathrm{C}(5)$ in $64 \%$ yield. The spectral data and optical rotation of alkaloid $(-)-5\left(\right.$ observed $[\alpha]_{\mathrm{D}}^{23}=-108(c=0.28, \mathrm{MeOH}) ;$ lit: $[\alpha]_{\mathrm{D}}=$ $\left.-30(c=0.038, \mathrm{MeOH})^{1 \mathrm{~b}}\right)$ were in agreement with literature values. Importantly, analysis of the common precursor (-)-45 by single-crystal X-ray diffraction unambiguously confirms the relative and absolute stereochemical configuration of all known $N 1^{\prime}$-sorbyl communesin alkaloids (-)-4, (-)-5, and (+)-6 for the first time.

We next proceeded with the first total synthesis of (-)-communesin G (7) and H (8). Rearrangement of key intermediate $(+)-19$ under the standard conditions followed by acylation with propionic anhydride efficiently furnished (-)-N8'-SES-communesin G (47) in $86 \%$ yield. Subsequent desulfonylation with TASF then afforded (-)-communesin G (7) in $74 \%$ yield, with spectral data and optical rotation (observed $[\alpha]_{\mathrm{D}}^{23}=-163(c=0.20, \mathrm{MeOH})$; lit: $[\alpha]_{\mathrm{D}}{ }^{25}=$ $\left.-157(c=0.021, \mathrm{MeOH})^{1 \mathrm{e}}\right)$ fully consistent with those reported in the isolation report. Similarly, rearrangement of heterodimer (+)-19, acylation with butyric anhydride, and desulfonylation of the intermediate heptacycle (-)-48 efficiently furnished (-)-communesin $\mathrm{H}(8)$ in $76 \%$ overall yield, with all spectral data and optical rotation (observed $[\alpha]_{\mathrm{D}}{ }^{23}=-168(c=0.38, \mathrm{MeOH})$; lit: $[\alpha]_{\mathrm{D}}{ }^{25}=-167(c=$ $0.024, \mathrm{MeOH})^{1 \mathrm{e}}$ ) identical to those available from isolation reports.

Finally, we turned our attention to the preparation of the reported structure of (-)-communesin I (9), the most recently isolated member of the communesin family. In order to introduce the $\left(3^{\prime \prime} S\right)$-hydroxy amide at $\mathrm{N1}^{\prime}$, we opted to use aldol addition product (+)-49 as the acyl donor ${ }^{13}$ after the key aminal reorganization. Rearrangement of heterodimer (+)-19 followed by acylation of the resulting communesin core with excess aldol adduct (+)-49 furnished amide (-)-50 in 84\% yield. Desulfonylation with TASF then afforded $(-)-\left(3^{\prime \prime} S\right)$ communesin I (9) in $86 \%$ yield, which enabled careful analysis of all spectral data and conclusive comparisons with the isolation data originally reported by Fan and co-workers ${ }^{1 \mathrm{f}}$ for natural (-)-communesin I. The ${ }^{1} \mathrm{H}$ and ${ }^{13} \mathrm{C}$ NMR signals associated with the core of the alkaloid were in good agreement with the isolation report, however key ${ }^{1} \mathrm{H}$ and ${ }^{13} \mathrm{C}$ signals on the acyl chain deviated notably from the expected values. Specifically, the ${ }^{13} \mathrm{C}$ NMR chemical shifts of C2" $\left(41.1^{13}\right.$ vs $\left.42.1^{1 \mathrm{f}} \mathrm{ppm}\right), \mathrm{C} 3^{\prime \prime}\left(68.1^{13}\right.$ vs $\left.69.0^{1 \mathrm{f}} \mathrm{ppm}\right)$, and $\mathrm{C} 4^{\prime \prime}$ $\left(38.8^{13}\right.$ vs $\left.39.5^{1 \mathrm{f}} \mathrm{ppm}\right)$ were found to be the most divergent.

In light of these data, we hypothesized that the stereochemical configuration at $\mathrm{C} 3$ " had been incorrectly assigned in the isolation report. Given the ease with which the diastereomeric aldol addition product (+)-51 could be prepared, we opted to synthesize the corresponding $\left(3^{\prime \prime} R\right)$ derivative (10) to test our hypothesis. Reorganization of key intermediate $(+)-19$ followed by acylation with aldol addition product (+)-51 furnished $\left(3^{\prime \prime} R\right)$ derivative (-)-52 in $48 \%$ yield, which upon $\mathrm{N} 8^{\prime}$-desulfonylation afforded $(-)-\left(3^{\prime \prime} R\right)$ communesin I (10) in $78 \%$ yield. Importantly, all ${ }^{1} \mathrm{H}$ and ${ }^{13} \mathrm{C}$ NMR data of this alkaloid were in excellent agreement with those reported in Fan's isolation report ${ }^{1 \mathrm{f}}$ of $(-)$-communesin 
I. The optical rotation was also consistent with the reported data, albeit our synthetic sample had a higher value (observed $[\alpha]_{\mathrm{D}}{ }^{23}=-137(c=0.22, \mathrm{MeOH})$; lit: $[\alpha]_{\mathrm{D}}{ }^{20}=-59(c=0.1$, $\left.\mathrm{MeOH})^{1 \mathrm{f}}\right)$. As a result, we herein reassign the stereochemical configuration at $\mathrm{C}^{\prime \prime}$ of this new communesin alkaloid from $(S)$ to $(R)$. This important finding validates the importance of our strategic late-stage $\mathrm{N}^{\prime}$ acylation, which enables the rapid diversification and functional derivatization of the communesin core.

To demonstrate the versatility of our highly convergent synthetic approach to all communesin alkaloids and related derivatives, we applied our synthetic strategy to prepare a constitutional isomer of the communesin skeleton, namely the iso-communesin ${ }^{5 \mathrm{~b}}$ core 17 (Scheme 1). We anticipated that this unnatural polycyclic topology would be accessible via an analogous aminal reorganization of a $\mathrm{C} 3 \mathrm{a}-\mathrm{C} 3 \mathrm{a}^{\prime}$ linked heterodimer containing a cyclotryptamine fragment with an inverted N1'/N8' substitution pattern. Treatment of this hypothetical substrate with the same basic conditions required to reorganize $(+)-19$ should result in the selective cleavage of the $\mathrm{C} 8 \mathrm{a}^{\prime}-\mathrm{N1}^{\prime}$ aminal, thereby resulting in the elements of the iso-communesin core after formation of the $\mathrm{C} 8 \mathrm{a}-\mathrm{N1}^{\prime}$ and $\mathrm{C} 8 \mathrm{a}^{\prime}-\mathrm{N} 1$ aminal linkages. As depicted in Scheme 8, fragment

\section{Scheme 8. Guided Rearrangement to an Unnatural} Communesin Related Topology ${ }^{a}$

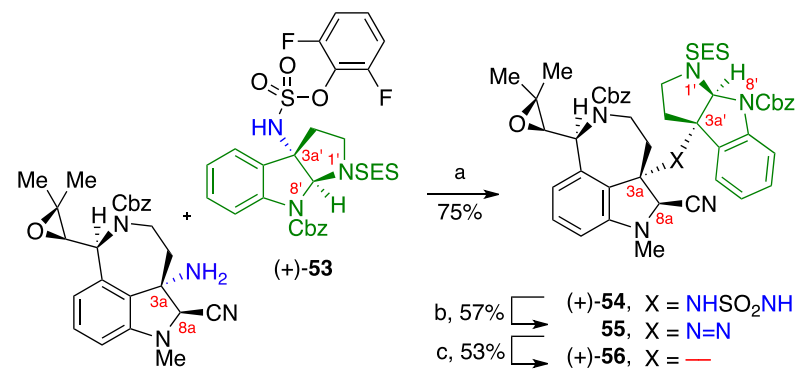

$(+)-38$

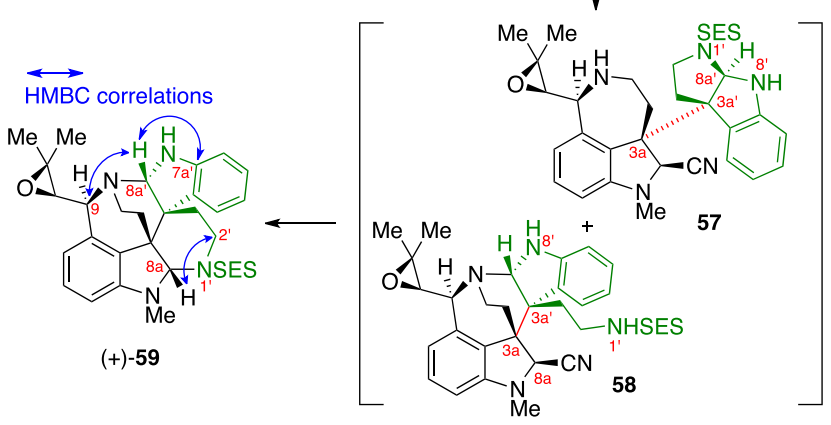

${ }^{a}$ Reagents and conditions: (a) DMAP, THF, $23{ }^{\circ} \mathrm{C}$; (b) polystyreneBEMP, N-chloro-N-methylbenzamide, $\mathrm{MeOH}, 23{ }^{\circ} \mathrm{C}$; (c) $h \nu$ (350 $\mathrm{nm}), 25{ }^{\circ} \mathrm{C}$; (d) (i) $\mathrm{H}_{2}, \mathrm{Pd}(\mathrm{OH})_{2} / \mathrm{C}, \mathrm{EtOH}, 23{ }^{\circ} \mathrm{C}$; (ii) $t$-BuOLi, EtOH, $60{ }^{\circ} \mathrm{C}$.

assembly of aminonitrile $(+)-38^{32}$ and the appropriately substituted $\mathrm{C} 3 \mathrm{a}^{\prime}$-sulfamate $(+)-53^{13}$ afforded sulfamide $(+)-54$ in $75 \%$ yield. Oxidation of $(+)-54$ under the same conditions employed for sulfamide $(+)-\mathbf{4 0}$ afforded the sensitive diazene $\mathbf{5 5}$ in $\mathbf{5 7 \%}$ yield. Photochemical irradiation of the diazene as a neat thin film at $350 \mathrm{~nm}$ then furnished the $\mathrm{C} 3 \mathrm{a}-\mathrm{C} 3 \mathrm{a}^{\prime}$ fused heterodimer $(+)-56$ in $53 \%$ yield. Hydrogenolysis of heterodimer (+)-56 resulted in formation of the anticipated heterodimeric diamine $\mathbf{5 7}$ along with the partially rearranged compound $\mathbf{5 8}(\mathbf{5 7 : 5 8 ,} 3: 1)$. Notably, when a pure sample of diamine $\mathbf{5 7}$ was treated with lithium tert-butoxide (10 equiv) in methanol- $d_{4}$ at $23{ }^{\circ} \mathrm{C}$, rapid and complete conversion to $\mathbf{5 8}$ was observed by ${ }^{1} \mathrm{H}$ NMR analysis. Evidently, the lower $\mathrm{p} K_{\mathrm{a}}$ of indoline $\mathrm{N} 8^{\prime} \mathrm{H}$ in heterodimer 57 relative to pyrrolidine $\mathrm{N}^{\prime} \mathrm{H}$ in (+)-19 enables cyclotryptamine-aminal opening even under the hydrogenolysis reaction conditions. Treatment of the crude hydrogenolysis product mixture of $\mathbf{5 7}$ and 58 with lithium tert-butoxide in ethanol at $60{ }^{\circ} \mathrm{C}$ resulted in clean conversion to iso-communesin derivative (+)-59 in $46 \%$ overall yield from $(+)-56$. The structural assignment of the unnatural polycyclic topology of alkaloid $(+)-59$ is supported by key HMBC data including the observed correlations $\mathrm{C} 8 \mathrm{aH}-\mathrm{C}_{2}^{\prime}$ and $\mathrm{C} 8 \mathrm{a}^{\prime} \mathrm{H}-\mathrm{C} 9$ that conclusively establish the presence of the $\mathrm{C} 8 \mathrm{a}-\mathrm{N}^{\prime}{ }^{\prime}$ and $\mathrm{C} 8 \mathrm{a}^{\prime}-\mathrm{N} 1$ aminals, respectively. The successful implementation of our synthetic strategy for the preparation of iso-communesin $(+)-59$ further highlights the generality of our guided heterodimer rearrangement utilized in our syntheses described above.

With samples of all known communesin alkaloids and a selection of unnatural derivatives in hand, we set out to probe the anticancer activity for this entire class of natural products. While previous isolation reports have evaluated the activity of selected natural communesins, no comprehensive comparison of the entire class of alkaloids across multiple cell lines has been performed. ${ }^{1}$ To this end, our synthetic samples of all nine naturally occurring communesins, a selection of seven complex intermediates from our synthetic campaign, and the $N 8^{\prime}$ sulfonylated iso-communesin (+)-59 were examined for cytotoxicity against human lung carcinoma (A549), prostate carcinoma (DU 145), colorectal carcinoma (HCT 116), cervical adenocarcinoma (HeLa), and breast adenocarcinoma (MCF7) cell lines. ${ }^{13}$ As depicted in Table $1,(-)$-communesin B (4) exhibited the highest potency of all the natural alkaloids tested across all cell lines, which is consistent with leukemiafocused assays performed in early isolation reports. ${ }^{1 \mathrm{a}, \mathrm{b}}$ The cytotoxicity of the natural products in our assays against adherent cell lines representing solid tumors is slightly less than those performed against the leukemia cell lines. This observation is not surprising given that it has been previously observed in large scale cell line profiling experiments that cell lines grown in suspension, such as leukemia and lymphoma, can be generally more sensitive to cytotoxic compounds relative to adherent cell lines. ${ }^{33}$ The next most active natural alkaloid, (-)-communesin C (5), exhibited an approximately 2 -fold decrease in potency in our assays, whereas alkaloids $(-)-2,(-)-3,(-)-7,(-)-9$, and (+)-43 were principally inactive across the cell lines examined.

Interestingly, advanced synthetic intermediates that contain an $N 8^{\prime}$-SES substituent generally exhibited a dramatic increase in potency relative to the $\mathrm{N}^{\prime}$ unsubstituted natural products (Table 1). For example, $N 8^{\prime}$-SES-communesin G, (-)-47, was found to exhibit an approximately 10-fold increase in potency relative to (-)-communesin $G(7)$. This increase in activity was found to hold irrespective of N8 substitution (e.g., (+)-46 vs (+)-6) or $\mathrm{N1}^{\prime}$ substitution (e.g., (-)-45 vs (-)-4). In this preliminary structure-activity relationship study, we also noted that the N8 substituent exerts a small but measurable influence on potency. For example, a two- to three-fold decrease in activity was observed moving from N8-methyl alkaloid (-)-4 to either N8-H alkaloid (-)-5 or N8-formyl alkaloid (+)-6. Additionally, we observed a general correlation between the size of the $\mathrm{N}^{\prime}$ substituent and the potency of the 
Table 1. Assessment of All Known Communesin Alkaloids and a Selection of Unnatural Derivatives for Cytotoxicity Against Five Human Carcinoma Cell Lines. ${ }^{a}$

\begin{tabular}{|c|c|c|c|c|c|c|c|c|c|}
\hline \multirow{2}{*}{ Compound } & \multirow{2}{*}{ Communesin } & \multirow{2}{*}{ N1' } & \multirow{2}{*}{ N8 } & \multirow{2}{*}{ N8' } & \multicolumn{5}{|c|}{$\mathrm{IC}_{50}(\mu \mathrm{M})$} \\
\hline & & & & & A549 & DU 145 & HCT 116 & HeLa & MCF7 \\
\hline \multicolumn{10}{|c|}{ SES Derivatives } \\
\hline$(-)-45$ & $N 8^{\prime}$-SES-B & Sorbyl & $\mathrm{Me}$ & SES & 19 & 24 & $>250$ & $>125$ & 5 \\
\hline$(-)-47$ & $N 8^{\prime}-$ SES-G & Propionyl & $\mathrm{Me}$ & SES & 19 & 17 & 18 & 16 & 8 \\
\hline$(-)-48$ & $(3 " S)-N 8$ '-SES-I & $(S)$-3-pentanol & $\mathrm{Me}$ & SES & 24 & 36 & 32 & 17 & 15 \\
\hline$(+)-46$ & $N 8^{\prime}$-SES-D & Sorbyl & $\mathrm{CHO}$ & SES & 39 & 29 & 27 & 15 & 24 \\
\hline$(-)-42$ & $N 8^{\prime}$-SES-A & Acetyl & $\mathrm{Me}$ & SES & 34 & 53 & 31 & 35 & 30 \\
\hline$(+)-59$ & iso-communesin & SES & $\mathrm{Me}$ & $\mathrm{H}$ & 335 & $>125$ & $>62.5$ & 64 & 58 \\
\hline \multicolumn{10}{|c|}{ N8'-H Natural Products and Derivatives } \\
\hline$(-)-4$ & $\mathrm{~B}$ & Sorbyl & $\mathrm{Me}$ & $\mathrm{H}$ & 56 & 45 & 60 & 47 & 34 \\
\hline$(-)-5$ & $\mathrm{C}$ & Sorbyl & $\mathrm{H}$ & $\mathrm{H}$ & 115 & 82 & 106 & 94 & 65 \\
\hline$(-)-1$ & $\mathrm{~F}$ & Acetyl & $\mathrm{Me}$ & $\mathrm{H}$ & 117 & 119 & $>125$ & 115 & 84 \\
\hline$(-)-10$ & $(3 " R)-\mathrm{I}$ (natural) & $(R)$-3-pentanol & $\mathrm{Me}$ & $\mathrm{H}$ & 120 & $>125$ & $>125$ & 120 & 90 \\
\hline$(-)-8$ & $\mathrm{H}$ & Butyryl & $\mathrm{Me}$ & $\mathrm{H}$ & $>125$ & $>125$ & 109 & 82 & 90 \\
\hline$(+)-6$ & $\mathrm{D}$ & Sorbyl & $\mathrm{CHO}$ & $\mathrm{H}$ & $>125$ & 84 & 111 & 63 & $>125$ \\
\hline$(-)-9$ & (3"S)-I (unnatural) & $(S)$-3-pentanol & $\mathrm{Me}$ & $\mathrm{H}$ & $>125$ & $>125$ & $>125$ & $>125$ & $>250$ \\
\hline$(-)-7$ & $\mathrm{G}$ & Propionyl & $\mathrm{Me}$ & $\mathrm{H}$ & $>125$ & $>125$ & $>125$ & $>125$ & $>250$ \\
\hline$(-)-2$ & $\mathrm{~A}$ & Acetyl & $\mathrm{Me}$ & $\mathrm{H}$ & $>125$ & $>250$ & $>250$ & $>125$ & $>250$ \\
\hline$(+)-43$ & $N 8$-formyl-E & Acetyl & $\mathrm{CHO}$ & $\mathrm{H}$ & $>250$ & $>250$ & $>250$ & $>125$ & $>250$ \\
\hline$(-)-3$ & $\mathrm{E}$ & Acetyl & $\mathrm{H}$ & $\mathrm{H}$ & $>500$ & $>500$ & $>500$ & $>500$ & $>500$ \\
\hline
\end{tabular}

${ }^{a}$ Cytotoxicity $\mathrm{IC}_{50}$ values (in $\mu \mathrm{M}$ ) after $72 \mathrm{~h}$ of compound treatment as determined by Cell Titer-Glo (Promega) which measures ATP levels as a surrogate for cell viability. Error is standard deviation of the mean, $n \geq 2 ; \mathrm{IC}_{50}=$ half maximal inhibitory concentration; Human Carcinoma Cell Lines: Lung (A549), Prostate (DU 145), Colorectal (HCT 116), Cervical (HeLa), and Breast (MCF7).

compound. This is particularly evident in the natural series, where the activity generally follows the trend $\mathrm{N1}^{\prime}$-sorbyl > pentan-3R-ol $>$ butyryl $>$ propionyl $>$ acetyl. As noted with the N8 substituent, the $N 8^{\prime}$-SES derivatives also followed the same general trend, but they were less sensitive to variation at this position. Lastly, the iso-communesin derivative (+)-59 exhibited inferior activity to all $\mathrm{N} 8$ '-SES communesin derivatives tested as well as a number of more modestly active $\mathrm{N} 8$ '-unsubstituted natural products.

Taken together, these preliminary data allow for the first side-by-side comparative analysis of a collection of communesin derivatives, including all nine naturally occurring communesins A-I (Figure 1), suggest primarily that (a) substitution at $\mathrm{N} 8$ ' can have a dramatic effect on potency; (b) N8-methyl derivatives exhibit improved activity relative to their N8-formyl or N8-unsubstituted counterparts; and (c) activity is nominally proportional to the size of the $\mathrm{N}^{\prime}$ substituent.

\section{CONCLUSIONS}

In summary, we have developed a unified enantioselective total synthesis of all known epoxide-containing communesin alkaloids (Figure 1), including the first total synthesis of communesins $\mathrm{C}-\mathrm{E}$, and $\mathrm{G}-\mathrm{I}$, in addition to related derivatives from a single advanced synthetic intermediate (+)-19. Our synthesis is predicated on the convergent and modular diazene-directed assembly of two complex fragments to secure the critical C3aC3a' linkage followed by a guided biomimetic aminal reorganization to deliver the heptacyclic core of these alkaloids. Concise gram-scale enantioselective syntheses of the fragments were devised, with highlights including the application of a new, specifically designed sulfinamide chiral auxiliary, an efficient calcium trifluoromethanesulfonate promoted intramolecular amination, a diastereoselective epoxidation that simultaneously converts the new chiral auxiliary to a sulfonylbased amine protective group, and the application of a silvermediated cyclotryptamine-C $3 \mathrm{a}^{\prime}$-sulfamate synthesis from a readily available enantioenriched $\mathrm{C} 3 \mathrm{a}^{\prime}$-bromocyclotryptamine. The modularity of our convergent approach enabled the stereochemical revision of (-)-communesin I (Figure 1), the most recently isolated communesin analogue. Furthermore, the generality of our biomimetic heterodimer reorganization was demonstrated in the synthesis of an unnatural constitutional isomer of the communesin skeleton, the iso-communesin ${ }^{5 b}$ derivative (+)-59. We also disclose the first side-by-side anticancer profiling of all nine naturally occurring communesin alkaloids and a selection of eight complex derivatives for cytotoxicity against five human cancer cell lines. From these data, we have identified (-)-communesin $\mathrm{B}$ as the most potent natural isolate and discovered that derivatives containing an $\mathrm{N} 8^{\prime}$-SES substituent exhibit up to a 10 -fold increase in potency over the natural products, with (-)-N8'-SES-communesins $\mathrm{B}$ (45) and $G$ (47) being the most potent communesin derivatives found to date. These findings form the basis of our ongoing efforts aimed at detailed chemical and biological study of these fascinating alkaloids.

\section{ASSOCIATED CONTENT}

\section{Supporting Information}

(PDF) The Supporting Information is available free of charge on the ACS Publications website at DOI: 10.1021/ jacs.9b07397.

Experimental procedures, spectroscopic data, and copies of NMR spectra (PDF) 
Crystallographic data (CIF)

Crystallographic data (CIF)

Crystallographic data (CIF)

\section{AUTHOR INFORMATION}

\section{Corresponding Author}

*movassag@mit.edu

\section{ORCID $\odot$}

Matthew M. Pompeo: 0000-0001-7761-085X

Mohammad Movassaghi: 0000-0003-3080-1063

Notes

The authors declare no competing financial interest.

\section{ACKNOWLEDGMENTS}

We acknowledge financial support from NIH-NIGMS (GM089732). M.M.P. acknowledges the Natural Sciences and Engineering Research Council of Canada (NSERC) for a PGS D3 Scholarship. We thank the Koch Institute Swanson Biotechnology Center for technical support, specifically the High Throughput Sciences Core. This work was supported in part by the Koch Institute Support (core) Grant P30-CA14051 from the National Cancer Institute. We thank Mr. Kyan A. D’Angelo, Dr. Charlene Tsay, and Dr. Peter Müller for assistance with X-ray crystal structure analysis of (-)-36, (-)-42, and (-)-45. We are grateful to Dr. Petra Lindovská, Dr. Olga Mukhina, and Mr. Kyan A. D’Angelo for helpful discussions.

\section{REFERENCES}

(1) (a) Numata, A.; Takahashi, C.; Ito, Y.; Takada, T.; Kawai, K.; Usami, Y.; Matsumura, E.; Imachi, M.; Ito, T.; Hasegawa, T. Communesins, Cytotoxic Metabolites of a Fungus Isolated From a Marine Alga. Tetrahedron Lett. 1993, 34, 2355-2358. (b) Jadulco, R.; Edrada, R. A.; Ebel, R.; Berg, A.; Schaumann, K.; Wray, V.; Steube, K.; Proksch, P. New Communesin Derivatives from the Fungus Penicillium sp. Derived from the Mediterranean Sponge Axinella verrucosa. J. Nat. Prod. 2004, 67, 78-81. (c) Hayashi, H.; Matsumoto, H.; Akiyama, K. New Insecticidal Compounds, Communesins C, D and E, from Penicillium expansum Link MK-57. Biosci., Biotechnol., Biochem. 2004, 68, 753-756. (d) Andersen, B.; Smedsgaard, J.; Frisvad, J. C. Penicillium expansum: Consistent Production of Patulin, Chaetoglobosins, and Other Secondary Metabolites in Culture and Their Natural Occurrence in Fruit Products. J. Agric. Food Chem. 2004, 52, 2421-2428. (e) Dalsgaard, P. W.; Blunt, J. W.; Munro, M. H. G.; Frisvad, J. C.; Christophersen, C. Communesins G and H, New Alkaloids from the Psychrotolerant Fungus Penicillium rivulum. J. Nat. Prod. 2005, 68, 258-261. (f) Fan, Y.-Q.; Li, P.-H.; Chao, Y.-X.; Chen, H.; Du, N.; He, Q.-X.; Liu, K.-C. Alkaloids with Cardiovascular Effects from the Marine-Derived Penicillium expansum Y32. Mar. Drugs 2015, 13, 6489-6504. (g) For the structurally related perophoramidine, see: Verbitski, S. M.; Mayne, C. L.; Davis, R. A.; Concepcion, G. P.; Ireland, C. M. Isolation, Structure Determination, and Biological Activity of a Novel Alkaloid, Perophoramidine, from the Philippine Ascidian Perophora namei. J. Org. Chem. 2002, 67, $7124-7126$.

(2) (a) Yang, J.; Wu, H.; Shen, L.; Qin, Y. Total Synthesis of (士)-Communesin F. J. Am. Chem. Soc. 2007, 129, 13794-13795. (b) Liu, P.; Seo, J. H.; Weinreb, S. M. Total Synthesis of the Polycyclic Fungal Metabolite ( \pm )-Communesin F. Angew. Chem., Int. Ed. 2010, 49, 2000-2003. (c) Belmar, J.; Funk, R. L. Total Synthesis of $( \pm)$-Communesin $\mathrm{F}$ via a Cycloaddition with Indol-2-one. J. Am. Chem. Soc. 2012, 134, 16941-16943. (d) Han, S.-J.; Vogt, F.; Krishnan, S.; May, J. A.; Gatti, M.; Virgil, S. C.; Stoltz, B. M. A Diastereodivergent Synthetic Strategy for the Syntheses of Communesin F and Perophoramidine. Org. Lett. 2014, 16, 3316-3319. (e) Han, S.-J.; Vogt, F.; May, J. A.; Krishnan, S.; Gatti, M.; Virgil, S. C.; Stoltz, B. M. Evolution of a Unified, Stereodivergent Approach to the Synthesis of Communesin F and Perophoramidine. J. Org. Chem. 2015, 80, 528-547. (f) For a review, see: Trost, B. M.; Osipov, M. Recent Advances on the Total Synthesis of Communesin Alkaloids and Perophoramidine. Chem. - Eur. J. 2015, 21, 16318-16343.

(3) (a) Zuo, Z.; Xie, W.; Ma, D. Total Synthesis and Absolute Stereochemical Assignment of (-)-Communesin F. J. Am. Chem. Soc. 2010, 132, 13226-13228. (b) Lathrop, S. P.; Pompeo, M.; Chang, W.-T. T.; Movassaghi, M. Convergent and Biomimetic Enantioselective Total Synthesis of (-)-Communesin F. J. Am. Chem. Soc. 2016, 138, 7763-7769. (c) Liang, X.; Zhang, T.-Y.; Zeng, X.-Y.; Zheng, Y.; Wei, K.; Yang, Y.-R. Ir-Catalyzed Asymmetric Total Synthesis of (-)-Communesin F. J. Am. Chem. Soc. 2017, 139, 33643367. (d) Park, J.; Jean, A.; Chen, D. Y.-K. Asymmetric Total Synthesis of Communesin F and a Putative Member of the Communesin Family. Angew. Chem., Int. Ed. 2017, 56, 1423714240. (e) Park, J.; Jean, A.; Chen, D. Y.-K. Organocatalytic and LateStage CH-Functionalization Enabled Asymmetric Synthesis of Communesin F and Putative Communesins. J. Org. Chem. 2018, 83, 6936-6957.

(4) Zuo, Z.; Ma, D. Enantioselective Total Syntheses of Communesins A and B. Angew. Chem., Int. Ed. 2011, 50, 1200812011.

(5) (a) Lin, H.-C.; Chiou, G.; Chooi, Y.-H.; McMahon, T. C.; Xu, W.; Garg, N. K.; Tang, Y. Elucidation of the Concise Biosynthetic Pathway of the Communesin Indole Alkaloids. Angew. Chem., Int. Ed. 2015, 54, 3004-3007. (b) Lin, H.-C.; McMahon, T. C.; Patel, A.; Corsello, M.; Simon, A.; Xu, W.; Zhao, M.; Houk, K. N.; Garg, N. K.; Tang, Y. P450-Mediated Coupling of Indole Fragments To Forge Communesin and Unnatural Isomers. J. Am. Chem. Soc. 2016, 138, 4002-4005.

(6) (a) Movassaghi, M.; Ahmad, O. K.; Lathrop, S. P. Directed Heterodimerization: Stereocontrolled Assembly via Solvent-Caged Unsymmetrical Diazene Fragmentation. J. Am. Chem. Soc. 2011, 133, 13002-13005. (b) Lathrop, S. P.; Kim, J.; Movassaghi, M. Radicalmediated Dimerization and Oxidation Reactions for the Synthesis of Complex Alkaloids. Chimia 2012, 66, 389-393. (c) Lathrop, S. P.; Movassaghi, M. Application of diazene-directed fragment assembly to the total synthesis and stereochemical assignment of (+)-desmethylmeso-chimonanthine and related heterodimeric alkaloids. Chem. Sci. 2014, 5, 333-340. (d) Lindovska, P.; Movassaghi, M. Concise Synthesis of (-)-Hodgkinsine, (-)-Calycosidine, (-)-Hodgkinsine B, $(-)$-Quadrigemine $\mathrm{C}$, and $(-)$-Psycholeine via Convergent and Directed Modular Assembly of Cyclotryptamines. J. Am. Chem. Soc. 2017, 139, 17590-17596.

(7) Xie, W.; Jiang, G.; Liu, H.; Hu, J.; Pan, X.; Zhang, H.; Wan, X.; Lai, Y.; Ma, D. Highly Enantioselective Bromocyclization of Tryptamines and Its Application in the Synthesis of (-)-Chimonanthine. Angew. Chem., Int. Ed. 2013, 52, 12924-12927.

(8) The stereochemistry of the epoxide is also implicated in the observed rate of decomposition. For example, we have found derivatives containing a (10S)-epoxide to be much more susceptible to this intramolecular opening than the corresponding (10R)derivatives.

(9) For reviews on the synthesis of and asymmetric additions to $\mathrm{N}$ tert-butanesulfinyl imines, see: (a) Ellman, J. A.; Owens, T. D.; Tang, T. P. N-tert-Butanesulfinyl Imines: Versatile Intermediates for the Asymmetric Synthesis of Amines. Acc. Chem. Res. 2002, 35, 984-995. (b) Ferreira, F.; Botuha, C.; Chemla, F.; Pérez-Luna, A. tertButanesulfinimines: structure, synthesis and synthetic applications. Chem. Soc. Rev. 2009, 38, 1162-1186. (c) Robak, M. T.; Herbage, M. A.; Ellman, J. A. Synthesis and Applications of tert-Butanesulfinamide. Chem. Rev. 2010, 110, 3600-3740. For general reviews of asymmetric additions to $N$-sulfinyl imines, see: (d) Davis, F. A.; Chen, B.-C. Asymmetric synthesis of amino acids using sulfinimines (thiooxime S-oxides). Chem. Soc. Rev. 1998, 27, 13-18. (e) Zhou, P.; Chen, B.-C.; Davis, F. A. Recent advances in asymmetric reactions using sulfinimines ( $\mathrm{N}$-sulfinyl imines). Tetrahedron 2004, 60, 8003- 
8030. (f) Senanayake, C. H.; Krishnamurthy, D.; Lu, Z.-H.; Han, Z.; Gallou, I. Enantiopure Sulfoxides and Sulfinamides: Recent Developments in Their Stereoselective Synthesis and Applications to Asymmetric Synthesis. Aldrichimica Acta 2006, 37, 93-104. (g) Morton, D.; Stockman, R. A. Chiral non-racemic sulfinimines: versatile reagents for asymmetric synthesis. Tetrahedron 2006, 62, 8869-8905.

(10) Sun, P.; Weinreb, S. M.; Shang, M. tert-Butylsulfonyl (Bus), a New Protecting Group for Amines. J. Org. Chem. 1997, 62, 86048608.

(11) (a) Weinreb, S. M.; Demko, D. M.; Lessen, T. A.; Demers, J. P. $\beta$-Trimethylsilylethanesulfonyl chloride (SES-Cl): A new reagent for protection of amines. Tetrahedron Lett. 1986, 27, 2099-2102. (b) Ribière, P.; Declerck, V.; Martinez, J.; Lamaty, F. 2(Trimethylsilyl)ethanesulfonyl (or SES) Group in Amine Protection and Activation. Chem. Rev. 2006, 106, 2249-2269.

(12) For relevant studies on the use of (-)-diacetone-D-glucose (DAG) as a chiral controller for the preparation of chiral sulfoxides and sulfinamides, see: (a) Llera, J. M.; Fernández, I.; Alcudia, F. An efficient synthesis of both enantiomers of chiral non racemic methylsulfoxides from DAG. Tetrahedron Lett. 1991, 32, 72997302. (b) Fernández, I.; Khiar, N.; Llera, J. M.; Alcudia, F. Asymmetric synthesis of alkane- and arenesulfinates of diacetone-dglucose (DAG): an improved and general route to both enantiomerically pure sulfoxides. J. Org. Chem. 1992, 57, 6789-6796. (c) Khiar, N.; Fernández, I.; Alcudia, F. Asymmetric synthesis of optically pure tert-butyl sulfoxides using the "DAG methodology. Tetrahedron Lett. 1994, 35, 5719-5722. (d) Fernández, I.; Valdivia, V.; Khiar, N. NIsopropylsulfinylimines as Useful Intermediates in the Synthesis of Chiral Amines: Expeditive Asymmetric Synthesis of the Calcimimetic (+)-NPS R-568. J. Org. Chem. 2008, 73, 745-748. (e) Chelouan, A.; Recio, R.; Alcudia, A.; Khiar, N.; Fernández, I. DMAP-Catalysed Sulfinylation of Diacetone-d-Glucose: Improved Method for the Synthesis of Enantiopure tert-Butyl Sulfoxides and tert-Butanesulfinamides. Eur. J. Org. Chem. 2014, 2014, 6935-6944.

(13) See the SI for details.

(14) The use of silver(I) carbonate as base was essential to provide the desired coupled product in high yield. A Heck protocol employing potassium carbonate as the base under otherwise identical conditions resulted in $81 \%$ yield of the protodebromination product with only $12 \%$ yield of the desired styrene.

(15) Harrington, P. J.; Hegedus, L. S.; McDaniel, K. F. PalladiumCatalyzed Reactions in the Synthesis of 3- and 4-Substituted Indoles. 2. Total Synthesis of the $N$-Acetyl Methyl Ester of $( \pm)$-Clavicipitic Acids. J. Am. Chem. Soc. 1987, 109, 4335-4338.

(16) Attempts to sequester the acid with mild inorganic bases such as sodium bicarbonate resulted in an equimolar amount of desired product relative to the catalyst loading, indicating that the acid is essential for catalyst turnover. For a proposed reaction mechanism, see: Banfi, L.; Basso, A.; Cerulli, V.; Guanti, G.; Riva, R. Polyfunctionalized Pyrrolidines by Ugi Multicomponent Reaction Followed by Palladium-Mediated $\mathrm{S}_{\mathrm{N}} 2^{\prime}$ Cyclizations. J. Org. Chem. 2008, 73, 1608-1611.

(17) Wakayama, M.; Ellman, J. A. Recycling the tert-Butanesulfinyl Group in the Synthesis of Amines Using tert-Butanesulfinamide. J. Org. Chem. 2009, 74, 2646-2650.

(18) Magnesium(II) perchlorate and magnesium(II) trifluoromethanesulfonate were also found to be competent promoters of the allylic amination, however due to safety considerations in the first case and slightly lower efficiency in the second case, we elected to proceed with calcium(II) trifluoromethanesulfonate.

(19) For alkene epoxidations mediated by catalytic amounts of 1,1,1trifluoroacetone with aqueous hydrogen peroxide as the primary oxidant, see: Shu, L.; Shi, Y. An Efficient Ketone-Catalyzed Epoxidation Using Hydrogen Peroxide as Oxidant. J. Org. Chem. 2000, 65, 8807-8810.

(20) For alkene epoxidations mediated by stoichiometric amounts of 1,1,1-trifluoroacetone, see: (a) Denmark, S. E.; Forbes, D. C.; Hays, D. S.; DePue, J. S.; Wilde, R. G. Catalytic Epoxidation of Alkenes with
Oxone. J. Org. Chem. 1995, 60, 1391-1407. (b) Yang, D.; Wong, M.K.; Yip, Y.-C. Epoxidation of Olefins Using Methyl(trifluoromethyl)dioxirane Generated in Situ. J. Org. Chem. 1995, 60, 3887-3889. (c) Denmark, S. E.; Wu, Z.; Crudden, C. M.; Matsuhashi, H. Catalytic Epoxidation of Alkenes with Oxone. 2. Fluoro Ketones. J. Org. Chem. 1997, 62, 8288-8289.

(21) Other common epoxidants also furnished (-)-33, albeit with slightly lower efficiency and diastereoselectivity. For example, exposure of a suspension of (-)-32 and sodium bicarbonate to meta-chloroperbenzoic acid (2.6 equiv) in dichloromethane provided $(-)-33$ in $65 \%$ yield and (-)-34 in 10\% yield.

(22) Matsumori, N.; Kaneno, D.; Murata, M.; Nakamura, H.; Tachibana, K. Stereochemical Determination of Acyclic Structures Based on Carbon-Proton Spin-Coupling Constants. A Method of Configuration Analysis for Natural Products. J. Org. Chem. 1999, 64, 866-876.

(23) The stereochemical configuration at C8a was determined by nuclear Overhauser effect analysis of a derivative.

(24) Scheidt, K. A.; Chen, H.; Follows, B. C.; Chemler, S. R.; Coffey, D. S.; Roush, W. R. Tris(dimethylamino)sulfonium Difluorotrimethylsilicate, a Mild Reagent for the Removal of Silicon Protecting Groups. J. Org. Chem. 1998, 63, 6436-6437.

(25) The addition of water was necessary to suppress intramolecular trapping of the C8a-iminium with the sulfamide.

(26) (a) Two disproportionation products account for the majority of the mass balance: the oxidized tetracyclic C3a-cyanoindole and the corresponding reduced $\mathrm{C}^{\prime} \mathrm{a}^{\prime}-\mathrm{H}$ cyclotryptamine. (b) In a related system, calculations suggest that the $\mathrm{C} 8 \mathrm{a}-\mathrm{CN}$ provides $3.8 \mathrm{kcal} / \mathrm{mol}$ in favor of the observed C3a-stereochemical outcome. See the Supporting Information in ref $3 \mathrm{~b}$.

(27) For in situ monitoring of the rearrangement by ${ }^{1} \mathrm{H}$ NMR in $\mathrm{CD}_{3} \mathrm{OD}$, see the SI.

(28) The average C-N1-C bond angle of $113.8^{\circ}$ and $114.1^{\circ}$ observed in the solid state structure of (-)-42 and (-)-45, respectively, demonstrates steric compression of the pyramidal tertiary amine.

(29) Deoxygenation suppresses the formation of minor side products derived from oxidation at the N8-methyl. For example, when (-)-45 was treated with TASF in nondegassed $N, N$ dimethylformamide, 5\% (+)-6 and $1 \%(-)-5$ were isolated in addition to $86 \%$ of $(-)-4$.

(30) For examples of chromium mediated oxidation of $N$-methyl amines to the corresponding formamides, see: (a) Cavé, A.; Kan-Fan, C.; Potier, P.; Le Men, J.; Janot, M.-M. Oxydation de quelques amines tertiaires par l'anhydride chromique dans la pyridine. Tetrahedron 1967, 23, 4691-4696. (b) Corey, E. J.; Balanson, R. D. A Total Synthesis of ( \pm )-Porantherine. J. Am. Chem. Soc. 1974, 96, 65166517. (c) He, B.; Song, H.; Du, Y.; Qin, Y. Total Synthesis of (-)-Ardeemin. J. Org. Chem. 2009, 74, 298-304. (d) Wu, H.; Xue, F.; Xiao, X.; Qin, Y. Total Synthesis of (+)-Perophoramidine and Determination of the Absolute Configuration. J. Am. Chem. Soc. 2010, 132, 14052-14054.

(31) The specific rotation data described in Numata's 1993 isolation report (ref 1a) for (-)-communesin A (2) and (-)-communesin B (4) are $116^{\circ}$ and $84^{\circ}$ more positive, respectively, than the data reported for the same compounds in Hayashi's 2004 isolation report (ref 1c). Our data for both compounds is fully consistent with the Hayashi isolation report and with Ma's total synthesis report.

(32) In this illustrative example, we elected to use aminonitrile $(+)-38$ instead of oxindole $(-)-23$ to facilitate access to the desired sulfamide $(+)-54$.

(33) Basu, A.; Bodycombe, N. E.; Cheah, J. H.; Price, E. V.; Liu, K.; Schaefer, G. I.; Ebright, R. Y.; Stewart, M. L.; Ito, D.; Wang, S.; Bracha, A. L.; Liefeld, T.; Wawer, M.; Gilbert, J. C.; Wilson, A. J.; Stransky, N.; Kryukov, G. V.; Dancik, V.; Barretina, J.; Garraway, L. A.; Hon, C. S.-Y.; Munoz, B.; Bittker, J. A.; Stockwell, B. R.; Khabele, D.; Stern, A. M.; Clemons, P. A.; Shamji, A. F.; Schreiber, S. L. An Interactive Resource to Identify Cancer Genetic and Lineage Dependencies Targeted by Small Molecules. Cell 2013, 154, 11511161. 